\title{
Alunos de Melhores Resultados Escolares Têm Estruturas Cognitivas de Menor Complexidade
}

\author{
Students with Better School Results Have Less Complex Cognitive \\ Structures
}

\author{
Sofia Maria Veríssimo Catarreira * \\ ORCID iD 0000-0002-8461-7619 \\ Luis Manuel Casas García** \\ ORCID iD 0000-0002-2752-1784 \\ Ricardo Luengo González*** \\ ORCID iD 0000-0003-4349-8606
}

\begin{abstract}
Resumo
Este artigo descreve uma experiência de aprendizagem que teve como objetivo estudar a relação da complexidade nas estruturas cognitivas dos alunos com os seus resultados escolares, cuja representação gráfica foi possível recorrendo à técnica Redes Associativas Pathfinder e ao software GOLUCA. A pesquisa envolveu 79 alunos com idades compreendidas entre os 14 e os 16 anos, que frequentavam o $9 .^{\circ}$ ano de escolaridade de escolas portuguesas. Numa fase inicial foi identificada a rede que melhor representava as relações entre os conceitos, num tema de geometria. Os alunos foram sujeitos a um teste de conhecimentos e a um teste GOLUCA antes e após a lecionação dos conteúdos e, deste modo, foi possível analisar e comparar a evolução das suas estruturas cognitivas face aos resultados escolares. Os resultados do nosso estudo sugerem que esta nova abordagem pode constituir um método útil para estudar e compreender as redes conceituais dos alunos durante os processos de aprendizagem dos conceitos matemáticos, ou outros, permitindo ao professor observar o progresso de aprendizagem do aluno de modo mais completo e, consequentemente, a realização de uma melhor adequação de tarefas e metodologias de ensino.
\end{abstract}

Palavras-chave: Teoria dos Conceitos Nucleares. Estrutura Cognitiva. Redes Associativas Pathfinder. Complexidade. GOLUCA.

\begin{abstract}
This article describes a learning experience with the aim at studying the complexity relationship between cognitive structures and school performances. Its graphic representation was made possible by using the Pathfinder Associative Networks technique and the GOLUCA software. This research involved 79 students with

\footnotetext{
* Doutora em Didácticas da Matemática, pela Universidade de Extremadura. (UNEX). Professora Adjunta Convidada no Politécnico de Portalegre(IPP), Portalegre, Portugal. Endereço para correspondência: Edifício Quartel do Trem, Avenida 14 de Janeiro, 7350-092 Elvas, Portugal.E-mail: sofiaverissimo@ gmail.com.

** Doutor em Psicopedagogia pela Universidade de Extremadura (UNEX). Professor Titular em Didácticas da Matemática na Universidade de Extremadura, Badajoz, Espanha. Endereço para correspondência: Facultad de Educación.Avda. de Elvas,s/n 06071 Badajoz (Espanha). E-mail: luisma@unex.es.

*** Doutor em Matemática, pela Universidade de Extremadura. (UNEX). Professor Catedrático na Universidade de Extremadura, Badajoz, Espanha. Endereço para correspondência: Facultad de Educación.Avda. de Elvas,s/n 06071 Badajoz (Espanha). E-mail: rluengo@unex.es.
} 
ages between 14 and 16 years old who attended the 9th grade of Portuguese schools. In a first approach, we identified the network that better represented the relations among the concepts in a geometry topic. Students did a knowledge test and a GOLUCA test before and after the topics were taught and, therefore, it was possible to analyze and compare the evolution of their cognitive structures having in mind their school performances. The results of our study suggest that this new approach may be part of an useful method to study and understand the conceptual networks of students during the process of learning Mathematics or other subjects' topics, allowing the teacher to watch the students' learning progress in a more complete way and consequently, accomplishing a better adequacy of tasks and teaching methods.

Keywords: Theory of Nuclear Concepts. Cognitive Structure. Pathfinder Associative Networks. Complexity. GOLUCA.

\section{Introdução}

\subsection{Estrutura cognitiva (aquisição e representação) e o processo de aprendizagem}

A mente humana despertou, ao longo dos tempos, uma enorme curiosidade por parte da comunidade científica. Desde cedo, se tentou compreender o seu funcionamento, o modo como se processa a aprendizagem e esta temática tem sido alvo de imensas investigações.

A aquisição de um novo conhecimento está condicionada por fatores externos, que são impulsionados pelo meio envolvente do sujeito e, simultaneamente, por fatores internos que são as representações prévias que o sujeito possui na sua estrutura cognitiva.

Segundo as teorias contemporâneas, a construção do conhecimento é um processo dinâmico, sendo que as vivências do sujeito influenciam o seu processo de aprendizagem. Atualmente pensa-se que o processo de aprendizagem de um determinado conhecimento tem lugar através do armazenamento organizado de informação na memória. A inserção de novos conhecimentos implica a reorganização das estruturas cognitivas, relacionando conceitos com outros já existentes.

Ausubel, Novak e Hanesian (1983) enfatizam que o processo de aprendizagem significativa é o mais importante no meio escolar. Todavia, para que isso ocorra, é necessário a conjugação de determinados requisitos básicos no processo de aprendizagem, ou seja, que o conteúdo ensinado seja relacionável com a estrutura cognitiva do aluno. Deste modo, o material institucional deve ser organizado de forma lógica, permitindo ao aluno a interação desses novos materiais com conceitos relevantes existentes na sua estrutura cognitiva e o tornem potencialmente significativo (AUSUBEL, NOVAK \& HANESIAN, 1983).

A estrutura cognitiva tem vindo a atrair progressivamente o interesse de diversos autores, sendo que muitos consideram que a mesma detém um papel relevante no processo de aprendizagem, filtrada por uma estrutura conceitual que permite a comunicação com os novos 
conhecimentos (AUSUBEL, NOVAK \& HANESIAN, 1983; CASAS \& LUENGO, 2004). O fator mais importante que influência a aprendizagem de um indivíduo é o conhecimento por ele adquirido com as suas vivências, devendo ser ensinado em função desse mesmo conhecimento (AUSUBEL, NOVAK \& HANESIAN, 1983).

O conhecimento da estrutura cognitiva do aluno constitui um ponto fundamental no processo de ensino-aprendizagem. Assim torna-se essencial para poder melhorar o processo educativo compreender como nela se integra e desenvolve um novo conhecimento.

A forma como se consolida o conhecimento na estrutura cognitiva de um aluno sobre um determinado conteúdo permite, na nossa opinião, que o professor promova um processo de ensino-aprendizagem mais eficaz e que, simultaneamente, promova maior sucesso educativo. Consideramos que é fundamental aliar o estudo da estrutura cognitiva com o processo de aprendizagem, permitindo uma visão mais completa de todo o processo. A caracterização do conhecimento do aluno sobre determinado conteúdo permite identificar as relações que se estabelecem entre os conceitos, podendo estar corretas ou não e, consequentemente, realizar uma intervenção educativa mais direcionada para fortalecer as relações consideradas fundamentais.

Atualmente existem diversos métodos que permitem a obtenção de dados da estrutura cognitiva do sujeito e a identificação e caracterização da organização do conhecimento do sujeito na estrutura cognitiva (CASAS \& LUENGO, 2002). A "pontuação de semelhança entre os conceitos" é outra técnica que pressupõe uma representação gráfica dos conceitos da memória. A representação é obtida através de valores numéricos que indicam a proximidade semântica entre os conceitos (GODINHO, LUENGO \& CASAS, 2007).

Com recursos a diversas técnicas, tais como Análise de Componentes Principais, Análise de Agrupamentos, Escalonamento Multidimensional e Redes Associativas Pathfinder (SCHVANEVELDT, 1989; CASAS, 2002; CASAS \& LUENGO, 2004) é possível a explicitação das relações de proximidade entre os conceitos, a representação e a análise dos dados obtidos. De um modo particular, as Redes Associativas Pathfinder_são sustentadas pela Teoria de Grafos, sendo obtidas com base num conjunto de características e as suas representações gráficas são constítuidas por nós/conceitos, entre os quais se estabelecem relações, e por arestas que descrevem a maior ou menor relação ou proximidades entre esses conceitos (CASAS \& LUENGO, 2004). Existem inúmeros pesquisadores que defendem a validade desta última técnica na representação da estrutura cognitiva (JONASSEN, BEISSNER \& YACCI, 1993; MCGAGHIE, 1996; DICERBO, 2007; LAU \& YUEN, 2009, 2010; TRUMPOWER \& SARWAR, 2010). 
A técnica Redes Associativas Pathfinder constitui uma possibilidade de caracterizar o conhecimento de um indivíduo sobre um determinado conteúdo, uma vez que a obtenção das representações visuais permite a análise do estado e evolução da estrutura cognitiva. De um modo particular, é possível identificar os conceitos que se destacam e as relações estabelecidas entre eles e, consequentemente, é possível condicionar o processo de aprendizagem em função dessa mesma estrutura. Esta caracterização do conhecimento das ligações estabelecidas entre os conceitos permite a elaboração de tarefas direcionadas que alteram a estrutura cognitiva, de modo a tornar a aprendizagem mais significativa e eficiente (FENKER, 1975; VERÍSSIMO, GODINHO, CASAS \& LUENGO, 2017; VERÍSSIMO, 2013; CASAS \& LUENGO, 2004).

O índice de similaridade das Redes Associativas Pathfinder pode ser obtido a partir da similaridade entre cada par de conceitos. As Redes Associativas Pathfinder, técnica referenciada pela Teoria dos Conceitos Nucleares, são determinadas aplicando o algoritmo Pathfinder (SCHVANEVELDT, 1989; CASAS \& LUENGO, 2002; 2004; KOUL, CLARIANA \& SALEHI, 2005) a partir de dados empíricos.

O método Pathfinder permite a representação da estrutura cognitiva recorrendo a algoritmos de representação gráfica de grafos, existindo programas informáticos que facilitam a recolha e representação gráfica da estrutura cognitiva, como é o caso do programa GOLUCA (GODINHO, LUENGO \& CASAS, 2007).

Na Figura 1 apresenta-se um exemplo de uma Rede Associativa Pathfinder em que se podem observar os seus constituintes, nomeadamente um conjunto de entidades ou nós, correspondendo aos conceitos, e um conjunto de ligações com um determinado peso entre os nós (SCHVANEVELDT, 1989; GODINHO, LUENGO \& CASAS, 2007; CASAS \& LUENGO, 2004). 


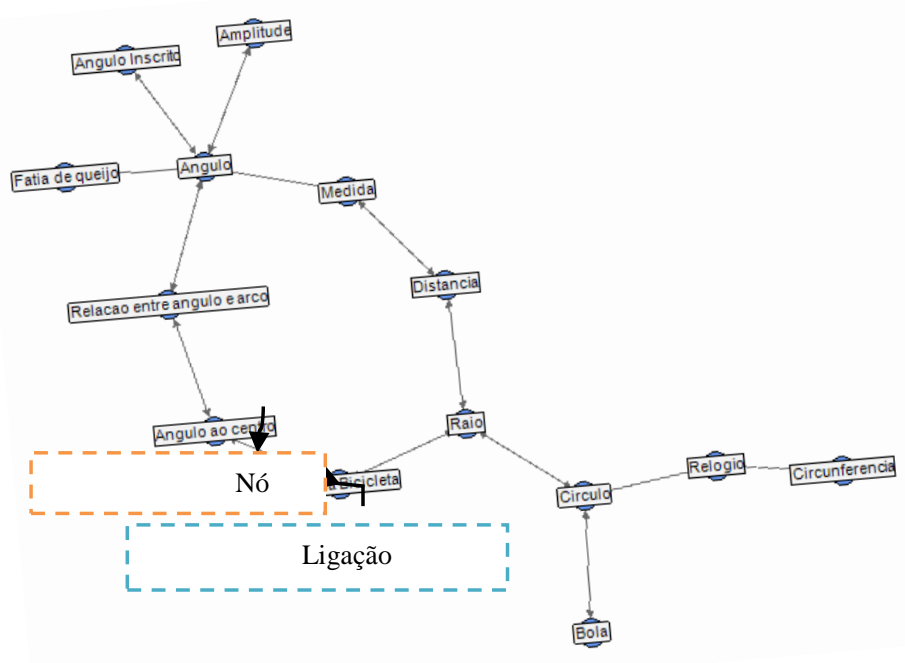

Figura 1 - Exemplo de uma Rede Associativa Pathfinder do programa GOLUCA Fonte: VERÍSSIMO, GODINHO, CASAS E LUENGO (2017, p. 7).

Atualmente existem várias áreas de investigação que recorreram às Redes Associativas Pathfinder, assumindo um papel importante, como é o caso de Educação, Medicina, Psicologia, Informática, Linguística entre outros.

\subsection{Unidades didáticas à luz da Teoria dos Conceitos Nucleares}

A Teoria dos Conceitos Nucleares (TCN) (CASAS \& LUENGO, 2004) emergiu da Ciência Cognitiva e fundamentou-se nas teorias relativas à organização do conhecimento humano, nomeadamente, nas teorias de Ausubel, Novak, entre outros. Esta teoria (TCN) defende os seguintes pressupostos:

-O conhecimento organiza-se a partir de pequenas unidades, inter-relacionadas em forma de rede, que formam a estrutura cognitiva.

-Os elementos que constituem as estruturas seguem uma correspondência funcional com os circuitos neuronais do cérebro humano e uma correspondência mental com as representações em forma de esquemas

- Cada conceito na mente equivale a uma estrutura, relativamente estável, com os elementos interrelacionados.

-Os conhecimentos prévios podem ser representados através dessas estruturas.

-A aprendizagem corresponde à modificação da estrutura cognitiva, por assimilação e reestruturação.

A TCN apresenta uma similitude com a interpretação de um mapa geográfico, em que 
um indivíduo, para identificar e conseguir deslocar-se de um local a outro, precisa passar por um conjunto de etapas. Inicialmente começa por observar o mapa que representa a zona de interesse e destaca alguns pontos que lhe chamam à atenção, ou porque conhece alguma localidade, ou algum nome lhe desperta o interesse, ou mesmo a disposição das marcas e das linhas que graficamente compõem o mapa.

Posteriormente, tenta delinear algumas rotas entre os pontos de partida e os de chegada. Esses percursos, numa fase inicial poderão resultar de tentativas, sem que o resultado seja o mais indicado para o objetivo final do indivíduo. Com o estudo em detalhe do mapa e outras informações que poderão fazer parte já do seu conhecimento, o indivíduo ganha uma “visão de conjunto"(CASAS \& LUENGO, 2004) e, a partir desse momento, conseguirá calcular a rota que melhor responde às suas necessidades.

No que concerne à aquisição de novo conhecimento, segundo a Teoria dos Conceitos Nucleares, o processo é análogo. Um indivíduo começará por tentar encontrar pontos de referência nas suas estruturas mentais. Numa fase seguinte, as estruturas reorganizar-se-ão de modo a encontrar percursos que relacionem os conceitos já existentes na sua estrutura cognitiva e passam a integrar os novos. O conhecimento é adquirido quando o indivíduo atingiu a "visão de conjunto" e assimilou os diversos percursos entre os conceitos da sua estrutura cognitiva, relacionada com o novo tema, com qual tinha sido confrontado. Apesar dos inúmeros percursos entre esses pontos de destaque o indivíduo escolherá aquele que, por diversos motivos, corresponde ao que lhe é mais significativo, logo com menos custos energéticos.

A TCN indica como principais elementos a "organização geográfica do conhecimento", defende que a aquisição do conhecimento segue um processo semelhante ao do conhecimento do ambiente físico que nos rodeia; os "conceitos nucleares", que servem de base à organização mental, não têm de ser necessariamente os mais gerais e/ou os mais abstratos; e os "caminhos de mínimo custo" são representações obtidas através das Redes Associativas Pathfinder que, para os sujeitos são as mais significativas, já que retiram delas o máximo benefício com o menor custo (CASAS \& LUENGO, 2004).

Segundo Casas e Luengo (2004), o nível de conhecimento sobre o processo de articulação de um novo conhecimento na estrutura cognitiva do aluno condiciona o sucesso da prática educativa. No Quadro 1 podemos observar de forma sucinta as principais diferenças entre a TCN e as teorias básicas anteriores. 


\begin{tabular}{|c|c|}
\hline Bases teóricas prévias & TCN \\
\hline Organização hierárquica do conhecimento & Organização geográfica do conhecimento \\
\hline Conceitos inclusores & Conceitos nucleares \\
\hline Complexidade crescente das estruturas cognitivas & Atalhos de mínimo custo \\
\hline Mapas Conceptuais & Redes Associativas Pathfinder \\
\hline
\end{tabular}

Quadro 1 - Relação TCN com as teorias prévias

Fonte: Elaborado pelos autores (2013).

O nosso estudo surge no seio da Teoria dos Conceitos Nucleares e tenta, através do estudo de estruturas cognitivas, perceber como se processa o conhecimento humano e como se comportam as estruturas cognitivas submetidas ao processo de aprendizagem. Neste âmbito recorremos à técnica Redes Associativas Pathfinder que nos permitem a descrição e visualização das estruturas cognitivas, antes e após o processo de ensino-aprendizagem e, consequentemente, estabelecemos a comparação entre elas.

Na TCN, com recurso à técnica Redes Associativas Pathfinder, é possível identificar os conceitos mais importantes em relação a um determinado tema, isto é, que se destacam nas estruturas cognitivas dos alunos. Assim, na elaboração da unidade didática, é possível criar uma sequência de ensino-aprendizagem inovadora. Esta toma como ponto de partida esses conceitos, denominados conceitos nucleares, e, através de tarefas direcionadas, reforça as relações estabelecidas entre eles consideradas corretas, elimina as incorretas e cria novas relações inexistentes consideradas importantes. Os objetivos principais desta nova modalidade de unidade didática têm por base os pressupostos da TCN, visam fornecer ferramentas que, por um lado, promovam a aprendizagem dos conteúdos programáticos e, por outro, permitam a aproximação da estrutura cognitiva do aluno daquela que é considerada mais correta por parte do professor (VERÍSSIMO, 2013).

Numa unidade didática tradicional os conceitos matemáticos apresentados do programa seguem a sequência dos mais gerais para os mais específicos. Alternativamente, nesta nova proposta de unidade didática, a ordem da apresentação das atividades, bem como a abordagem dos conteúdos são determinados pelo carácter mais ou menos importante desses conceitos na estrutura cognitiva prévia dos alunos, avaliada inicialmente.

Assim, ao longo da implementação da unidade de ensino, a seleção das tarefas, bem como a abordagem dos conteúdos partem dos conceitos nucleares e do objetivo (eliminar/criar/reforçar) de cada ligação estabelecida entre os conceitos que se pretende abordar, nunca descuidando os conteúdos programáticos a atingir. Uma tarefa não tem de ser necessariamente diferente das usualmente propostas na unidade didática tradicional, mas a sua 
implementação estará sempre aliada a esses dois objetivos. Por exemplo, um conceito nuclear, num determinado tema de geometria, poderá ser "relógio" e a abordagem do tema terá como ponto de partida esse conceito, podendo iniciar por explorar a sua comprensão, bem como todas as relações estabelecidas com os outros conceitos que se destacam no tema. As tarefas, problemas e exercícios usuais são selecionados com base nessas mesmas relações, sempre aliados ao objetivo de elimina-la, cria-la ou mantê-la. Procede-se de igual modo com os outros conceitos nucleares e abordam-se todos os conteúdos programáticos do tema, numa sequência programática totalmente inovadora.

Segundo a TCN, a complexidade da estrutura cognitiva de um indivíduo tende a diminuir em função do aumento de idade e do grau de "maturidade" do conhecimento. Defende que o aumento do nível de conhecimento numa determinada área implica que sejam privilegiadas determinadas ligações entre os conceitos (atalhos de mínimo custo), sendo essas mais utilizadas pelo indivíduo, o que torna a sua estrutura cognitiva mais simples.

\subsection{Estrutura cognitiva e o conceito de complexidade de redes}

O conceito de complexidade a que dedicamos especial atenção nesta investigação é um indicador quantitativo das Redes Associativas Pathfinder. Teve a sua origem em trabalhos desenvolvidos por Casas e Luengo (CASAS, 2002; CASAS \& LUENGO, 2004), que partiram de recomendações de Novak para a avaliação de mapas conceituais. O índice de complexidade relaciona diversas características das referidas redes, nomeadamente a densidade da rede (quociente entre o número de ligações do grafo e o total de ligações possíveis), o número de nós múltiplos (nós com duas os mais ligações) e o grau dos nós múltiplos (intensidade da relação dos nós múltiplos com os restantes nós).

Este indicador permite avaliar quantitativamente a complexidade das distintas Redes Associativas Pathfinder (CASAS, 2002) e assume valores entre 0 e 1, sendo que, quanto maior for o seu valor, maior é a complexidade da rede em estudo.

Um índice de complexidade elevado é bastante notório numa simples visualização de uma Rede Associativa Pathfinder. Em forma de exemplo apresentamos duas redes, uma com uma complexidade elevada, rede A (Figura 2) comparativamente a outra, rede B (Figura 3). 


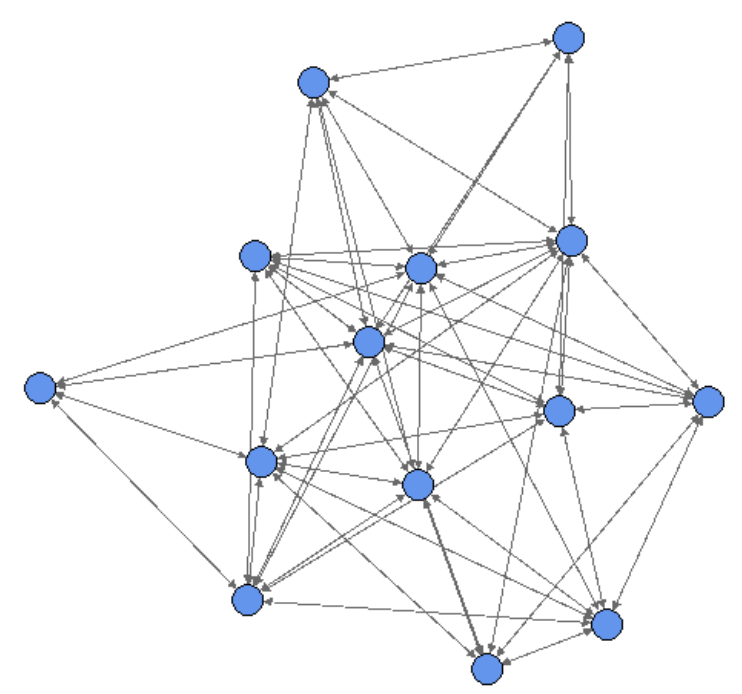

Figura 2 - Exemplo da rede Associativa Pathfinder A Fonte: VERÍSSIMO, LUENGO E CASAS (2016, p. 44)

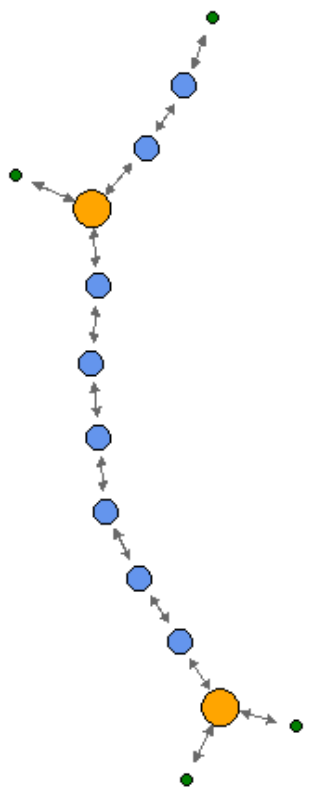

Figura 3 - Exemplo de uma Rede Associativa Pathfinder B Fonte: Elaborado pelos autores (2013).

A Rede Associativa Pathfinder da Figura 2 apresenta um índice de complexidade superior comparativamente à Figura 3, sendo que, para além de apresentar um maior número de ligações entre os conceitos, constata-se também que cada conceito está ligado a um maior número de conceitos.

Segundo a TCN, este índice de complexidade da estrutura cognitiva do aluno vai-se alterando ao longo do processo de aprendizagem. Segundo Casas e Luengo (2004), o aluno, quando é sujeito a um processo de aprendizagem numa determinada temática, destaca determinados conceitos e estabelece relações entre eles.

Apesar do aluno ter conhecimento de mais ligações, à medida que desenvolve um 
maior domínio, acaba por recorrer com maior frequência às relações mais simples e obtém valores de complexidade das Redes Associativas Pathfinder cada vez menores. Podemos afirmar que a estrutura cognitiva do aluno, segundo a TCN, simplifica-se com a aprendizagem, sendo que apenas se destacam nela as relações fundamentais entre os conceitos.

\section{Objetivos e questões de investigação}

Neste estudo estipulamos como principal objetivo perceber a relação existente entre os resultados escolares e o índice de complexidade de redes. Assim, colocamos a seguinte questão de investigação:

Após o decorrer de um processo de aprendizagem, os alunos com estruturas cognitivas de menor complexidade, medido através do índice de complexidade de redes, obtêm melhores resultados na prova de avaliação de conhecimentos?

\section{Metodologia}

Nesta investigação centramo-nos no tema de Geometria "Circunferência, Ângulo ao Centro e Ângulo Inscrito". Inicialmente desenvolvemos um estudo prévio que nos permitiu identificar os conceitos nucleares do tema e que consistiram no ponto de partida para a elaboração da unidade didática implementada. Para a determinação dos conceitos nucleares, que enumeramos no apartado 4.1, foi necessário realizar um trabalho exaustivo de pesquisa na bibliografia científica existente sobre tema e nos manuais escolares das diversas editoras, bem como realizar entrevistas com professores experientes.

Este estudo prévio envolveu 76 alunos com idades entre 14 e 16 anos, permitiu-nos identificar os conceitos matemáticos e as associações que os alunos estabelecem no tema, bem como as relações que estipulam entre eles. Estes alunos do estudo prévio foram entrevistados, realizaram antes e após a lecionação dos conteúdos em estudo, um teste de conhecimentos e outro teste que recorre às Redes Associativas Pathfinder, que permitiu caracterizar as redes das suas estruturas cognitivas, mais propriamente ao software GOLUCA, que mais à frente será detalhado.

Para o estudo principal foi elaborada uma unidade didática segundo os pressupostos a TCN, nomeadamente "organização geográfica do conhecimento"; "caminhos de mínimo custo" e "conceitos nucleares" que se encontra detalhado em Veríssimo (2013) e em 
Veríssimo, Godinho, Casas e Luengo (2014), sendo que, para esta investigação, iremos nos focar em apenas duas localidades das estudadas. Foi ainda adotado um processo de pesquisa composto por três fases: a fase de coleta de dados (pretest); fase de implementação da unidade de ensino e de aprendizagem; e por último a fase de recolha de dados (postest), como se encontra ilustrado na Figura 4.

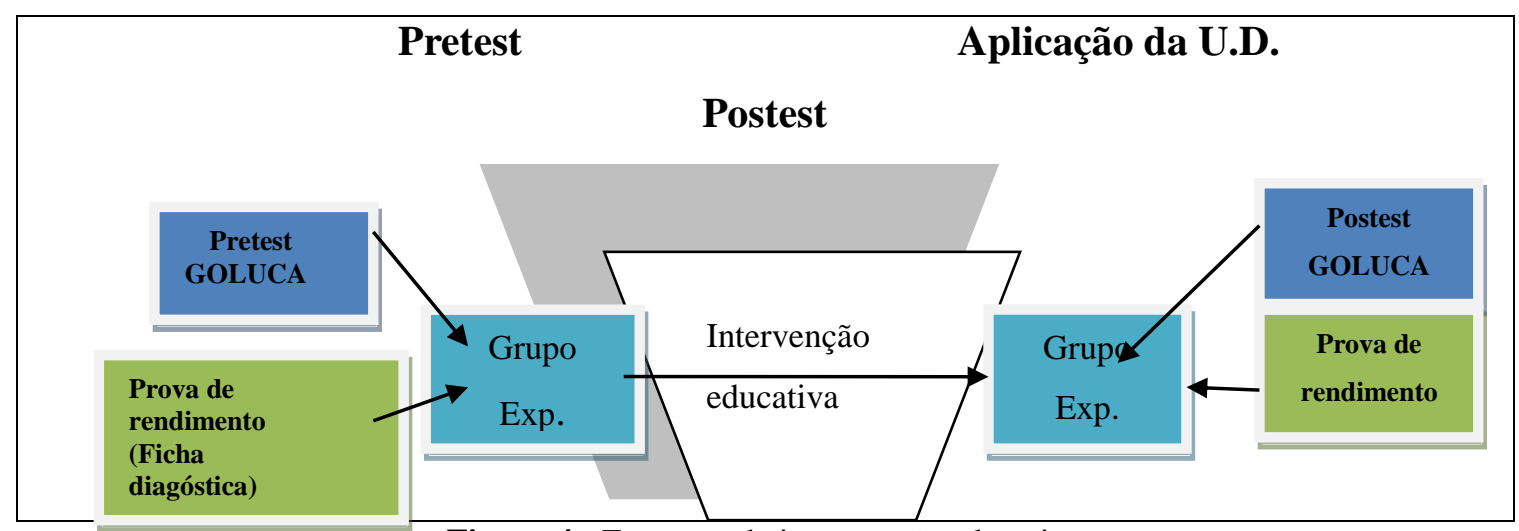

Figura 4 - Esquema da intervenção educativa Fonte: Elaborado pelos autores (2013).

No pretest os alunos foram sujeitos a uma prova de conhecimentos e a um teste GOLUCA e assim foi possível identificar as estruturas cognitivas iniciais relacionadas com o tema.

A segunda fase do estudo consistiu na implementação da unidade didática TCN. É importante referir que todos os alunos tiveram acesso às mesmas tarefas, bem como exercícios, problemas e tarefas de investigação. Decorreram diversas reuniões entre o pesquisador e os professores e, para além de aferir critérios de atuação homogéneos foram disponibilizados todos os materiais necessários para a investigação e implementação das unidades didáticas. Estas reuniões foram importantes, pois permitiram aos diversos professores das diferentes escolas envolvidas adquirir critérios de atuação idênticos e colocar todos os alunos em igualdade de circunstâncias no processo de aprendizagem.

Finalmente, no postest, os alunos realizaram novamente uma prova de conhecimentos que consistiu num conjunto de exercícios e problemas análogos ou retirados dos exames nacionais de $9 .^{\circ}$ ano, cotado na escala de 0 a 100 pontos percentuais. A prova inicial de conhecimentos denominamos de prova diagnóstico e o teste escrito de conhecimentos final denominamos de prova final. Realizaram ainda um postest GOLUCA e, deste modo, foi possível realizar a comparação dos resultados da fase inicial com a final.

\subsection{Amostra do estudo}


No que concerne ao estudo principal participaram duas escolas do Alto Alentejo de Portugal, envolvendo 79 alunos e 8 professores. A recolha da nossa amostra efetuou-se por conveniência geográfica, após um primeiro contato com diversas escolas que possuíam turmas no nível de ensino a que a nossa investigação se referia. No conjunto de escolas que aceitou colaborar connosco, foi escolhida uma amostra aleatória entre as diversas turmas que cada uma possuía.

\subsection{Recolha de dados}

A recolha de dados foi feita usando o software GOLUCA e decorreu em dois momentos: um que precedeu a implementação da unidade didática (pretest) e outro que ocorreu depois do processo de aprendizagem (postest). Este programa permite a recolha, representação gráfica e análise das estruturas cognitivas sob a forma de Redes Associativas Pathfinder.

A recolha dos dados no software GOLUCA, que se encontra detalhado em Godinho et al. (2007) começa com a identificação do aluno e em seguida é-lhe apresentada uma interface própria que avalia a similaridade entre os conceitos. Durante a recolha de dados, o software GOLUCA apresenta um par de conceitos e uma barra de pesos (Figura 5). O programa apresenta todos os pares de conceitos possíveis, escolhendo um par de cada vez e de modo aleatório. A barra de pesos corresponde aos valores que poderão ser atribuídos como peso entre dois conceitos. Neste processo o aluno clica sobre a barra de pesos atribuindo dessa forma a proximidade ou similitude entre dois conceitos. À medida que estabelece uma relação entre dois conceitos, o par de conceitos altera-se para o seguinte. 


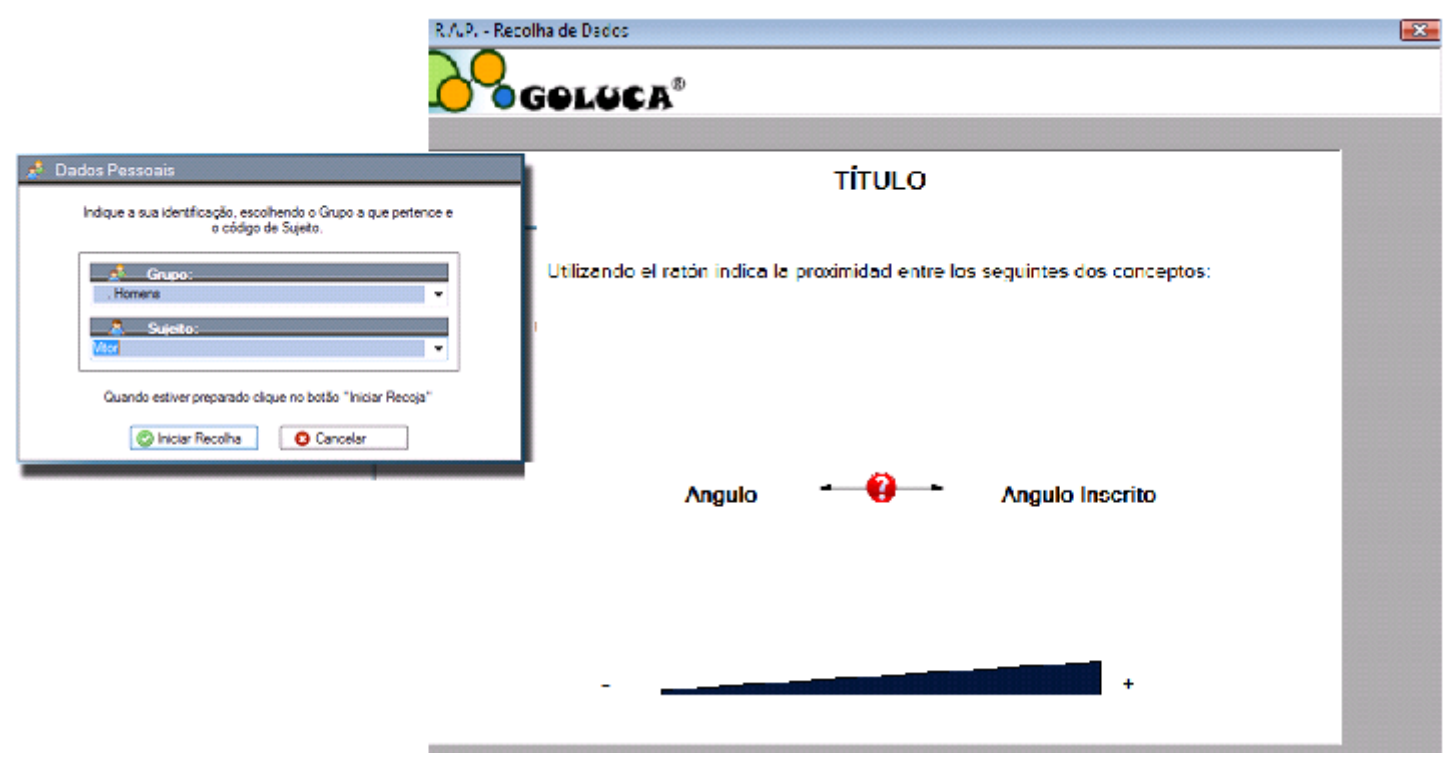

Figura 5 - Interface de identificação e recolha de dados Fonte: VERÍSSIMO (2013, p. 84).

Após a recolha dos dados, o software GOLUCA guarda-os sob a forma de uma matriz de similitude para posterior análise e representação gráfica sob a forma de Redes Associativas Pathfinder (Figura 6). Estas redes representam a estrutura cognitiva do aluno sob a forma de um grafo, cujas ligações correspondem às relações mais fortes entre os conceitos, estabelecidas pelo aluno, durante a fase de recolha de dados. Este programa permite-nos analisar a evolução de uma estrutura cognitiva de um indivíduo, visto que realiza a contagem e identificação de todas as ligações estabelecidas por cada sujeito. Estes dados são armazenados em bases de dados. Na Figura 6 pode-se observar o aspeto do interface do programa GOLUCA. 


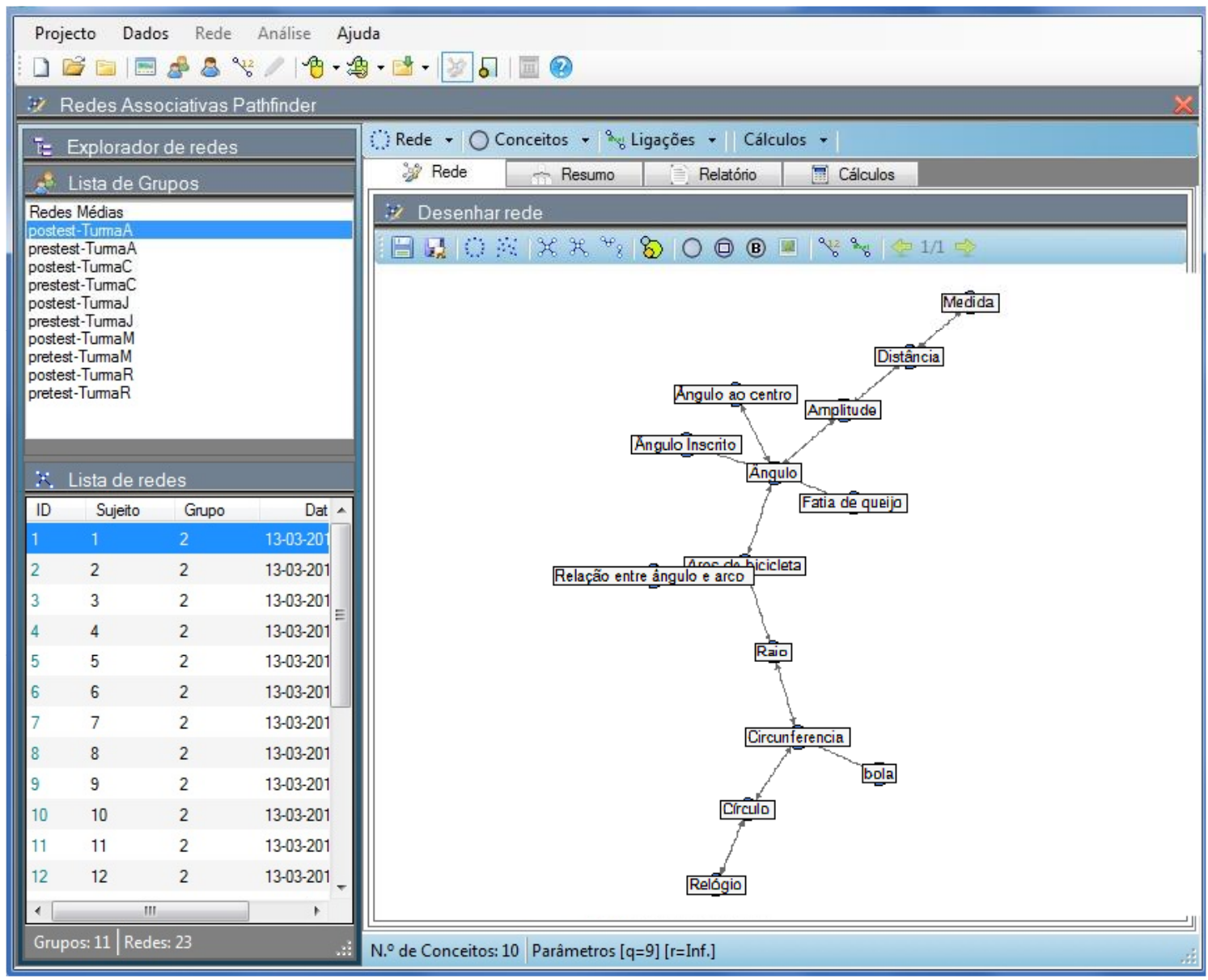

Figura 6- Interface da representação e análise de uma Rede Associativa Pathfinder Fonte: VERÍSSIMO $(2013,86)$.

O software GOLUCA permite a contagem das ligações estabelecidas entre os vários conceitos, bem como o índice de complexidade. Neste caso, os resultados que apresentamos dizem respeito ao total de ligações da amostra.

\section{Resultados}

\subsection{Resultados do estudo prévio}

O nosso estudo incidiu na temática da Circunferência, Ângulo ao Centro e Ângulo Inscrito.

O estudo prévio permitiu elaborar a lista de conceitos para aplicar o teste GOLUCA, a saber: Circunferência; Círculo, Raio, Bola; Aros de bicicleta; Fatia de queijo; Relação entre ângulo e arco; Ângulo; Ângulo ao Centro; Ângulo Inscrito; Amplitude; Distância e Medida. Nesta lista foram ainda identificados os conceitos mais importantes no tema, nomeadamente, Círculo, Circunferência, Raio, Ângulo, ou Ângulo Inscrito. No que diz respeito a associações 
e exemplos o que mais se destacaram foram bola ou fatia de queijo, respetivamente associados ao conceito de esfera e de setor circular (VERÍSSIMO, 2013).

Estes conceitos dizem respeito à "imagem de conceito" segundo Tall e Vinner (1981), sendo que, além de conceitos abstratos matemáticos, envolve objetos reais ou exemplos genéricos, tais como bola, relógio, aros de bicicleta ou fatia de queijo, que representam, respetivamente, esferas, ângulos, circunferências e setor circular. Na Figura 7, pode-se observar a rede de referência obtida para a elaboração da unidade didática (VERÍSSIMO, 2013)

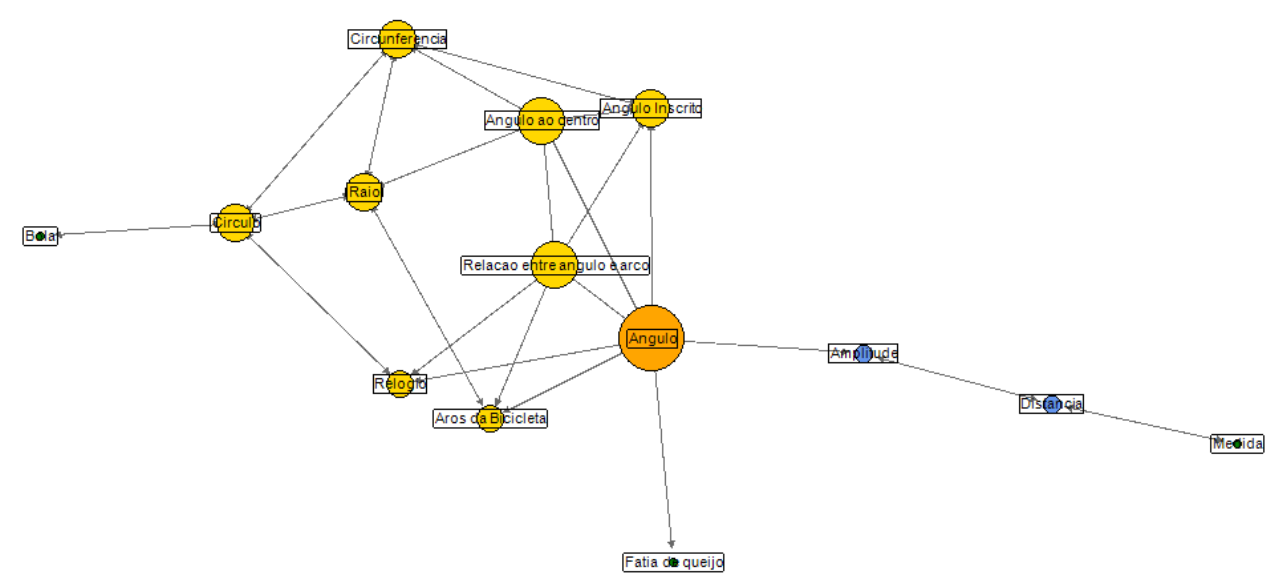

Figura 7 - Rede de referência Fonte: VERÍSSIMO (2013, p. 185).

Como se pode observar a rede permite identificar a ligações estabelecidas entre os conceitos e identificar claramente os conceitos nucleares e é através deles que todo o processo de ensino se desenvolve (VERÍSSIMO, 2013).

Apresentamos, ainda a título de exemplo, uma Rede Associativa Pathfinder de um aluno A do estudo, sendo que se pode constatar as diferenças entre o pretest (Figura 7) e postest (Figura 8) do teste GOLUCA. 


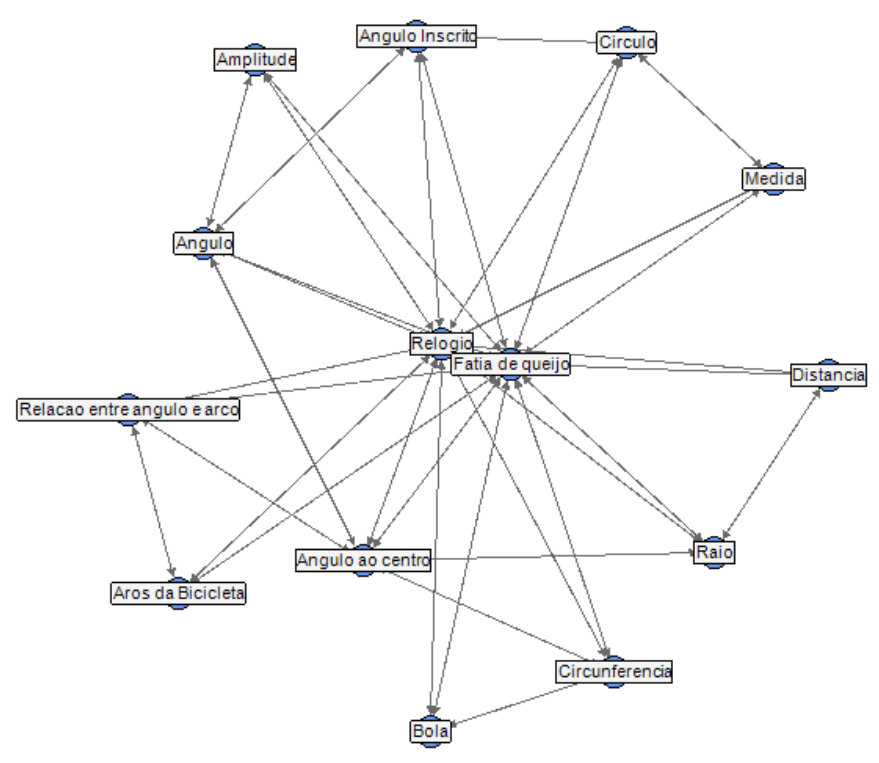

Figura 8 - Estrutura cognitiva do aluno A no pretest Fonte: Elaborado pelos autores (2013)

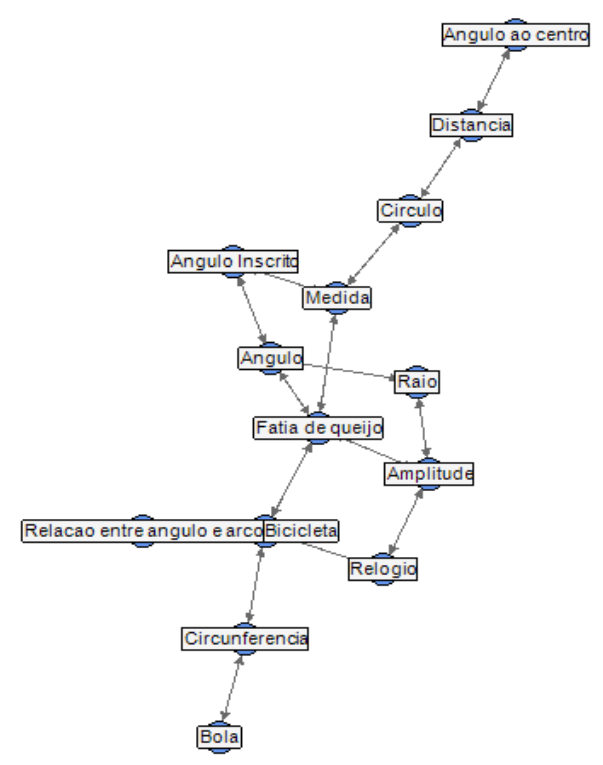

Figura 9 - Estrutura cognitiva do aluno A no postest Fonte: Elaborado pelos autores (2013).

Observando comparativamente as duas redes anteriores do mesmo aluno, facilmente se constata que o índice de complexidade diminuiu. Como se pode ver na Figura 9, o aluno evidencia um menor número de ligações entre os conceitos, comparativamente à Figura 8 . De um modo mais particular, o conceito de "círculo" possui no pretest quatro ligações e no postest apenas duas, o que de modo análogo ocorre com outros conceitos. Podemos ainda referir que no pretest o aluno apresenta um 0,1565 de índice de complexidade e no postest os valores desceram para 0,00587 .

\subsection{Resultados do estudo definitivo pretest-postest}


Após a obtenção das Redes Associativas Pathfinder dos alunos nas fases inicial e final, foi possível calcular o índice de complexidade de cada uma e, através do coeficiente de Pearson, realizar uma comparação entre elas. Os resultados apresentados dizem respeito aos alunos envolvidos no estudo principal. Foi ainda realizada uma comparação com os resultados obtidos nos testes escritos de conhecimentos (prova diagnóstico e prova final).

Tabela 1- Relação do índice de complexidade inicial e prova diagnóstico

\begin{tabular}{llll}
\hline & & prova_diagnóstico & pre_complexidade \\
\hline prova_diagnóstico & Correlação de Pearson & 1 &,- 071 \\
& Sig. (bilateral) & &, 533 \\
& $\mathrm{~N}$ & 79 & 79 \\
pre_complexidade & Correlação de Pearson &,- 071 & 1 \\
& Sig. (bilateral) &, 533 & \\
& $\mathrm{~N}$ & 79 & 79 \\
\hline
\end{tabular}
Fonte: VERISSIMO (2013).

$\mathrm{Na}$ análise do índice de complexidade, apresentados na Tabela 1, constatou-se que as Redes Associativas Pathfinder apresentaram uma correlação negativa fraca entre os resultados do pretest GOLUCA e as classificações obtidas na prova diagnóstico.

Tabela 2 - Índice de complexidade e resultados do teste de conhecimentos final

\begin{tabular}{llcc}
\hline & & pos_complexidade & prova_final \\
\hline pos_complexidade & Correlação de Pearson & 1 &,$- 254(*)$ \\
& Sig. (bilateral) & &, 024 \\
& $\mathrm{~N}$ & 79 & 79 \\
prova_final & Correlação de Pearson &,$- 254(*)$ & 1 \\
& Sig. (bilateral) &, 024 & 79 \\
& $\mathrm{~N} \quad 79$ & \\
& $*$ a correlação é significativa ao nível 0,05 (bilateral). & \\
\multicolumn{2}{c}{ Fonte: VERÍSSIMO (2013). }
\end{tabular}

$\mathrm{Na}$ análise do índice de complexidade do postest GOLUCA, face ao teste de conhecimentos realizado após a lecionação dos conteúdos, apresentados na Tabela 2, constatase uma correlação negativa moderada, pelo que os alunos com melhores resultados na prova de conhecimentos foram os que apresentavam um índice de complexidade menor e este resultado é estatisticamente significativo $(\mathrm{p}=0,024)$.

Tabela 3 - Resultados do teste diagnóstico e resultados do teste de conhecimentos final

\begin{tabular}{ccccc}
\hline & Media & N & Desvio tip. & Erro tip. da media \\
\hline prova_diagnóstico & 47,329 & 79 & 22,122 & 2,489 \\
prova_final & 63,658 & 79 & 18,503 & 2,082 \\
\hline
\end{tabular}

Fonte: VERÍSSIMO (2013).

$\mathrm{Na}$ análise da Tabela 3 pode-se observar que os alunos apresentam uma taxa de sucesso de 47,329\% na prova diagnóstico, com um desvio padrão em relação à média de 
$22,122 \%$, comparativamente à prova final de conhecimentos, na qual se constata uma taxa de sucesso de 63,658\%, com um desvio padrão em relação à média de 18,503\%. Constata-se assim um aumento dos resultados da prova diagnóstico para a prova final de 16,329\%.

Tabela 4 - Contraste de resultados da prova diagnóstico como a prova final de conhecimentos

\begin{tabular}{|c|c|c|c|c|c|c|c|}
\hline & \multicolumn{7}{|c|}{ prova_diagnóstico - prova_final } \\
\hline \multicolumn{5}{|c|}{ Diferenças relacionadas } & $\mathrm{t}$ & gl & Sig.(bilateral) \\
\hline \multirow[b]{2}{*}{ Média } & \multirow{2}{*}{$\begin{array}{c}\text { Desvio } \\
\text { típ. }\end{array}$} & \multirow{2}{*}{$\begin{array}{l}\text { Erro } \\
\text { típ. da } \\
\text { média } \\
\end{array}$} & \multicolumn{2}{|c|}{$\begin{array}{l}\text { 95\% Intervalo de } \\
\text { confiança para a } \\
\text { diferença }\end{array}$} & \multirow[b]{2}{*}{ Média } & \multirow{2}{*}{$\begin{array}{c}\text { Desvio } \\
\text { típ. }\end{array}$} & \multirow{2}{*}{$\begin{array}{l}\text { Erro típ. da } \\
\text { média }\end{array}$} \\
\hline & & & Superior & Inferior & & & \\
\hline 16,329 & 23,804 & 2,678 & $-21,661$ & $-10,997$ & $-6,097$ & 78 &, 000 \\
\hline
\end{tabular}

Numa fase inicial foi investigado o tipo de teste a aplicar (paramétrico ou não paramétrico), tendo sido aplicado um teste de Kolmogorov-Smirnov, um teste de sequências e teste de Levene da homogeneidade de variâncias. Após esta análise procedeu-se ao estudo da comparação da prova inicial e prova final do índice de complexidade. Na análise das Tabelas 3 e 4, constata-se que na comparação dos resultados do teste diagnóstico face à prova final de conhecimentos houve uma melhoria dos resultados escolares. Este resultado confirma que após a lecionação da unidade didática os alunos melhoraram os seus conhecimentos sobre o tema e este resultado é estatisticamente significativo $(\mathrm{p}=0,000)$.

\section{Discussão e conclusões do estudo}

O nosso trabalho incidiu sobre um tema de geometria, por ser um dos quais os alunos obtêm piores resultados escolares. Assim é fundamental perceber as alterações ou as características da estrutura cognitiva para que se possa realizar uma intervenção mais eficaz por parte do professor.

Através do conhecimento das relações estabelecidas entre os conceitos é possível perceber o grau de relevância de cada uma e identificar as que estão estabelecidas de forma incorreta e posteriormente realizar tarefas que aliem simultaneamente dois objetivos: por um lado acionem a aprendizagem dos conteúdos; e por outro promovam a alteração da estrutura cognitiva, para que as relações estabelecidas entre os conceitos sejam as corretas e as consideradas fundamentais.

De acordo com os objetivos estabelecidos no início da nossa investigação e com os 
resultados obtidos nas provas escritas inicial e final e na análise realizada às redes que apresentam as relações estabelecidas entre os conceitos, descritos na seção anterior, confirmamos que os alunos com estruturas cognitivas de menor complexidade foram os que obtiveram melhores resultados escolares, por sua vez existe uma correlação linear moderada entre os resultados escolares e o índice de complexidade das estruturas cognitivas dos alunos.

A técnica Redes Associativas Pathfinder permite-nos a identificação de forma clara e simples das ligações estabelecidas pelos alunos entre os conceitos, além de visualizar o aspecto das estruturas cognitivas dos alunos antes e após o processo de aprendizagem. Estas redes, usadas de forma adequada, constituem um método simples, rápido e eficaz para a identificação de conceitos nucleares em redor dos quais estrutura o conhecimento. Permitem, também, a exploração e o conhecimento das redes dos alunos, quando se encontram perante uma situação nova de ensino-aprendizagem.

O programa GOLUCA constitui, na nossa opinião, uma importante ferramenta metacognitiva que permitiu, de um modo simples, e não muito moroso, a obtenção das Redes Associativas Pathfinder dos alunos e os valores referentes a diversos parâmetros de possível análise. Existem vários estudos que recorrem a esta metodologia, tais como Carvalho, Ramos, Casas e Luengo (2010); Veríssimo, Casas, Luengo e Godinho (2011); Casas et al. (2013); Bizarro, Luengo, Casas e Carvalho (2015); Almeida, Casas e Luengo (2017), sendo que, na maioria destas investigações, a metodologia permitiu a comparação das alterações ocorridas na estrutura cognitivas antes e depois da realização da experiência. Podemos ainda destacar, como grande vantagem, a obtenção de dados quantitativos e de representações gráficas das estruturas cognitivas dos sujeitos em estudo.

$\mathrm{Na}$ nossa opinião, esta investigação destaca a importância do estudo das estruturas cognitivas para o processo de ensino e os possíveis condicionantes para as metodologias e recursos do mesmo.

Estes resultados inserem-se nos pressupostos da Teoria dos Conceitos Nucleares e reforça a importância do conhecimento da estrutura cognitiva no processo de ensinoaprendizagem.

\section{Referências}

ALMEIDA, C.; CASAS, L.; LUENGO, R.. Estudo da estrutura cognitiva dos alunos dos 9. ${ }^{\circ}$ (14-15 anos de idade) e $12 .^{\circ}$ anos (17-18 anos de idade) de escolaridade sobre o conceito deprobabilidade: $\mathrm{o}$ contributo das teorias dos conceitos nucleares e dos conceitos Threshold. Revista Latinoamericana de Investigación en Matemática Educativa, México, v. 20, n. 1, p. 9-38, 2017

AUSUBEL, D.P.; NOVAK, J.; HANESIAN, H. Psicología Educativa: un punto de vista cognoscitivo. 
México: Trillas. 1983.

BIZARRO, N. et al. Aplicación de las Redes Asociativas Pathfinder al análisis de los conceptos forma, tamaño y color en alumnos con Discapacidad Intelectual. Revista Lusófona de Educação, Portugal, v. 29, p. 121-144, 2015.

CARVALHO, J. et al. Estrutura cognitiva dos alunos e aprendizagem conceptual da Matemática: contributos para o seu conhecimento através da técnica de Redes Associativas Pathfinder. Educação, Formação \& Tecnologias, Portugal, v. 3, n. 1, p. 15-30, 2010.

CASAS, L. El estudio de la estructura cognitiva de alumnos a través de Redes Asociativas Pathfinder. Aplicaciones y posibilidades en Geometría. 2002. 385 f. Tese (Doutorado em Didácticas da Matemática) - Faculdade de Educação. Universidade de Extremadura, Espanha, 2002.

CASAS, L.; LUENGO, R. El estudio de la estructura cognitiva de alumnos através de Redes Asociativas Pathfinder. Aplicaciones y posibilidades en Geometría. Campo Abierto, Espanha, v. 16, p. 13-33, 2002.

CASAS, L.; LUENGO, R. Teoría de los Conceptos Nucleares: Aplicación en Didáctica de las Matemáticas, un ejemplo en Geometría. In: LUENGO, R. (Coord.). Líneas de Investigación en Educación Matemática. Badajoz: Sociedad Extremeña de Educación Matemática "Ventura Reyes Prósper”, 2004. p. 127-164.

CASAS, L. et al. Theory of Nuclear Concepts: A New Approach to Understand and Represent Cognitive Structures. In: PUMILLA, P. (Ed.). Handbook of Research on Didactic Strategies and Technologies for Education: Incorporating Advancements. United States of America: IGI Global, 2013. p. 591-604.

DICERBO, K. Knowledge Structures of Entering Computer Networking Students and Their Instructors. Journal of Information Technology Education, EUA, v. 6, p. 263-277, 2007.

KOUL, R.; CLARIANA, R.B.; SALEHI, R. Comparing several human and computer-based methods for scoring concept maps and essays. Journal of Educational Computing Research, EUA, v. 32, n. 3, p. 261-273, 2005.

FENKER, R. M. The organization of conceptual materials: A methodology for measuring ideal and actual cognitive structures. Instructional Science, Amsterdam, v. 4, p. 33-57, 1975.

GODINHO, V.; LUENGO, R.; CASAS, L. Implementação do software GOLUCA e aplicação à modificação de redes conceptuais. 2007. 306 f. Dissertação (Diploma de Estudos Avançados) Faculdade de Educação Universidade de Extremadura, Badajoz. 2007.

JONASSEN, D.; BEISSNER, K.; YACCI, M. Structural Knowledge: Techniques for Representing, Conveying and Acquiring Structural Knowledge. Hillsdale, NJ: Laurence Erlbaum Associates, 1993.

LAU, W.; YUEN, A. Predictive Validity of Measures of the Pathfinder Scaling Algorithm on Programming Performance: Alternative Assessment Strategy for Programming Education. Journal of Educational Computing Research, EUA, v. 41, n. 2, p. 227-250, 2009.

LAU, W.; YUEN, A. Promoting Conceptual Change of Learning Sorting Algorithm through the Diagnosis of Mental Models: THE EFFECTS OF GENDER AND LEARNING STYLES. IN Computers \& Education, EUA, v. 1, n. 54, p. 275-288, 2010.

MCGAGHIE, W. Comparison of Knowledge Structures with the Pathfinder Scaling Algorithm. Annual Meeting of the American Educational Research, New York, 1996. 
SCHVANEVELDT, R. Pathfinder Associative Networks. Studies in Knowledge Organization. Norwood, NJ: Ablex, 1989.

TALL, D.; VINNER, S. Concept image and concept definition in Mathematics with particular reference to limits and continuity. Educatinal Studies in Mathematics, Switzerland, v. 12, p. 151$169,1981$.

TRUMPOWER, D. L.; SARWAR, G. S. Effectiveness of structural feedback provided by Pathfinder networks. Journal of Educational Computing Research, EUA, v. 43, n. 1, p. 7-24, 2010.

VERÍSSIMO, S. et al. Teaching Unit on Geometry designed from the point of view of the Theory of Nuclear Concepts. The Future of Education, Florence, 2011.

VERÍSSIMO, S. et al. Theory of nuclear concepts: Its application in the mathematics classroom. In: ATEE, 4., 2012, Coimbra. Anais... Coimbra: PDTE, 2012.

VERÍSSIMO, S. et al. Identificação de conceitos prévios dos alunos recorrendo a métodos qualitativos. Indagatio Didactica, Portugal, v. 6, n. 3, p. 110-124, 2014.

VERÍSSIMO, S.; CASAS, L.; LUENGO. Unidades didáticas à luz da Teoria dos Conceitos NuclearesUm exemplo de Geometria. Campo Abierto, Espanha, v. 35, n. 2, p. 41-58, 2016.

VERÍSSIMO, S. A introdução das ideias da Teoria dos Conceitos Nucleares no ensino da Geometria e as suas implicações. 2013. 909 f. Tese (Doutorado em Didácticas de Matemática) Faculdade de Educação. Universidade de Extremadura, Espanha, 2013.

VERÍSSIMO, S. et al. Evaluation of Changes in Cognitive Structure after the Learning Process in Mathematics. International Journal of Innovation in Science and Mathematics Education, Australian, v. 25, n. 2, p. 17-33, 2017.

Submetido em 29 de Julho de 2018. Aprovado em 09 de Fevereiro de 2019. 